\title{
ZUR KONSTRUKTION PERIODISCHER LÖSUNGEN VON PSEUDODIFFERENTIALGLEICHUNGEN MIT HILFE VON OPERATORENALGEBREN
}

\author{
KARL DOPPEL und NIELS JACOB
}

\section{Einleitung}

1. Periodische Lösungen von elliptischen linearen partiellen Differentialgleichungen mit konstanten oder periodischen Koeffizienten wurden systematisch zuerst von P. D. Lax [6] untersucht, um mit Hilfe der erzielten Resultate gewisse Fragen über die Regularität der schwachen Lösungen von Randwertproblemen anzugreifen.

Diese Theorie ist von L. Bers und M. Schechter weitergeführt und vollendet worden. Sie haben die Ergebnisse in ihrem Übersichtsartikel über elliptische Differentialgleichungen [1] zusammengefaßt. Einige neuere Aspekte dazu findet man bei J. Eells [2]. In der Arbeit [8] untersuchten I. S. Louhivaara und C. G. Simader periodische Lösungen von gewissen ,,koerzitiven“ linearen partiellen Differentialgleichungen, die nicht notwendigerweise elliptisch zu sein brauchten.

In der vorliegenden Arbeit wird nun eine Methode angegeben (man vergleiche J. J. Kohn und L. Nirenberg [5]), die die Konstruktion einer Inversen bzw. einer verallgemeinerten Inversen von linearen partiellen Differentialoperatoren mit konstanten Koeffizienten in der Vordergrund treten läßt.

2. Es sei $Q$ ein ( $n$-dimensionaler) achsenparalleler Würfel mit Kantenlänge $2 \pi$ in $\boldsymbol{R}^{n}$. Mit $S$ bezeichnen wir die Menge der Funktionen $\varphi: \boldsymbol{R}^{n} \rightarrow \boldsymbol{C}$, die sich als endliche Summen

$$
\varphi(x)=(2 \pi)^{-n / 2} \sum_{\substack{k \in Z^{n} \\ \text { endl. }}} \varphi_{k} e^{i(k, x)}
$$

mit Fourierkoeffizienten $\varphi_{k} \in \boldsymbol{C}$ darstellen lassen. Für einen Differentialoperator $P(D)$ mit konstanten komplexen Koeffizienten und für ein vorgegebenes $\psi$ aus $S$ ist das Lösen der Gleichung

$$
P(D) \varphi=\psi
$$

in $S$ (wir sind zunächst nur an Lösungen $\varphi$ aus $S$ interessiert) äquivalent dem 
Lösen des (linearen) Gleichungssystems

$$
(P(D) \varphi)_{k}=P(k) \varphi_{k}=\psi_{k}
$$

mit den Fourierkoeffizienten $\varphi_{k}$ und $\psi_{k}=(P(D) \varphi)_{k}$ von $\varphi$ bzw. $\psi$. (Man vergleiche etwa Louhivaara-Simader [8], S. 90.)

In dem Falle, daß $P(k) \neq 0$ für alle $k \in Z^{n}$ ist, wird

$$
\varphi(x)=(2 \pi)^{-n / 2} \sum_{k \in Z^{n}} \frac{\psi_{k}}{P(k)} e^{i(k, x)}
$$

eine Lösung der Gleichung (0.2) bzw. (0.3) sein. Durch (0.4) ist aber auch der zu $P(D)$ inverse Operator $B(D)=(P(D))^{-1}$ mit $B(k)=1 / P(k)$ auf $S$ erklärt. Dieser ordnet jedem $\psi \in S$ die Funktion $\varphi$ aus (0.4) zu. Es jst klar, daß $B(D)$ kein Differentialoperator mehr ist. Daher erweitern wir die Klasse der zulässigen Operatoren und haben im Titel der Arbeit die dadurch erklärten Gleichungen Pseudodifferentialgleichungen genannt. Wir bemerken, daß diese Operatoren im allgemeinen keine Pseudodifferentialoperatoren sind, da sie nicht pseudolokal zu sein brauchen (man vergleiche [9]).

3. Man nehme nun anstelle eines Polynoms $P$ eine beliebige Abbildung $A: Z^{n} \rightarrow C$ und erkläre mit dieser Abbildung einen Operator $A(D): S \rightarrow S$ durch

$$
(A(D) \varphi)_{k}:=A(k) \varphi_{k} \text { für alle } k \in Z^{n} .
$$

Sei $\psi$ ein vorgegebenes Element aus $S$ mit den Fourierkoeffizienten $\psi_{k}$. Ein Element $\varphi \in S$ mit Fourierkoeffizienten $\varphi_{k}$, für welches das (lineare) Gleichungssystem

$$
A(k) \varphi_{k}=\psi_{k}
$$

erfüllt ist, nennen wir nun eine Lösung in $S$ der Operatorgleichung

$$
A(D) \varphi=\psi \text {. }
$$

Folglich hat die Gleichung (0.7) für ein festes $\psi \in S$ dann und nur dann eine Lösung in $S$, wenn $\psi_{k}=0$ gilt für jedes $k \in Z^{n}$ mit $A(k)=0$.

Ist $A(k) \neq 0$ für alle $k \in Z^{n}$, so besitzt (0.7) für jedes $\psi$ aus $S$ eine eindeutig bestimmte Lösung

$$
\varphi(x)=(2 \pi)^{-n / 2} \sum_{k \in Z^{n}} \frac{\psi_{k}}{A(k)} e^{i(k, x)} .
$$

(Man beachte, daß wegen $\psi \in S$ obige Summe endlich ist.)

Im Falle, da $\beta A(k)=0$ nur für endlich viele $k \in Z^{n}$ ist, kann man für die Gleichung (0.7) eine Fredholmsche Alternative zeigen: $\mathrm{Zu}$ einem $\psi \in S$ existiert dann und nur dann eine Lösung in $S$, wenn $\psi_{k}=0$ gilt für jedes $k \in Z^{n}$ mit $A(k)=0$. Diese Lösung ist eindeutig bestimmt bis auf ein beliebiges additives Element aus 
dem endlich dimensionalen Teilraum von $S$, der von der Menge

$$
\mathscr{N}^{\prime}:=\left\{\varphi \in S \mid \varphi_{k} \neq 0 \text { nur für jene } k \in Z^{n} \text { mit } A(k)=0\right\}
$$

erzeugt ist. Also existiert in diesem Falle zu $A(D)$ ein Operator $B(D): S \rightarrow S$ derart, daß

$$
B(D) \circ A(D)=I-R(D)
$$

mit einem ,,endlichen“ Operator $R(D)$ gilt, dessen Wertemenge mit $\mathscr{N}^{\prime}$ zusammenfällt. Der Operator $B(D)$ ist somit eine verallgemeinerte Inverse zu $A(D)$, und wir werden $B(D)$ eine Parametrix von $A(D)$ nennen.

4. Damit die Funktion auf der rechten Seite der Gleichung (0.7) auch außerhalb der Menge $S$ gewählt werden kann, werden wir $S$ in bezug auf geeignete Skalarprodukte vervollständigen, die Operatoren auf diese Hilberträume (stetig) fortsetzen und dann Gleichungen der Gestalt $(0.7)$ in den erhaltenen Hilberträumen $\mathrm{zu}$ lösen versuchen.

Beachtet man nun, daß in dem Ausdruck (0.8) einer Lösung von (0.7) im Nenner der Wert der Abbildung $A$ auftritt und nach der Vervollständigung von $S$ im allgemeinen unendlich viele Fourierkoeffizienten des Elements der rechten Seite von (0.7) von Null verschieden sein werden, sieht man die Notwendigkeit, die Konvergenz der Reihe (0.8) durch konvergenzerzeugende Faktoren zu erzwingen.

Aus diesem Grund wird zur beliebigen Funktion $\zeta: Z^{n} \rightarrow C$ eine Gewichtsfunktion $\zeta_{*}$ durch

$$
\zeta_{*}(k):=\left(1+|\zeta(k)|^{2}\right)^{1 / 2} \quad \text { für alle } \quad k \in Z^{n}
$$

erklärt. Wir betrachten dann die Menge aller Abbildungen $A: Z^{n} \rightarrow C$, für die mit einem festen $t^{\prime} \in \boldsymbol{R}$ und mit $C_{t^{\prime}, A} \in \boldsymbol{R}, C_{t^{\prime}, A}>0$ die Abschätzung

$$
|A(k)| \leqq C_{t^{\prime}, A} \zeta_{*}^{t^{\prime}}(k) \quad \text { für alle } \quad k \in Z^{n}
$$

gilt. Für die entsprechenden Operatoren $A(D): S \rightarrow S$ kann man nun in natürlicher Weise eine $\zeta_{*}$-Ordnung als das Infimum von allen zulässigen Potenzen $t^{\prime} \in \boldsymbol{R}$ definieren. Für $t \in \boldsymbol{R}$ erklären wir $\mathscr{A}^{t}\left(\zeta_{*}\right)$ als die Menge der Operatoren $A(D)$, deren $\zeta_{*}$-Ordnung kleiner oder gleich $t$ ist. Weiter schreiben wir

$$
\mathscr{A}^{-\infty}\left(\zeta_{*}\right):=\bigcap_{t \in R} \mathscr{A}^{t}\left(\zeta_{*}\right)
$$

und nennen die Operatoren aus dieser Klasse $\zeta_{*}$-glättend.

Durch Vervollständigung von $S$ in bezug auf das Skalarprodukt

$$
(\varphi, \psi)_{r, \zeta_{*}}=\sum_{k \in Z^{n}} \zeta_{*}^{2 r}(k) \bar{\varphi}_{k} \psi_{k}
$$

mit einem positiven $r \in \boldsymbol{R}$ erhält man nun den Hilbertraum $H_{\zeta_{*}}^{r}(Q)$. Es stellt sich dann heraus, daß die Operatoren aus $\mathscr{A}^{t}\left(\zeta_{*}\right)(t \in \boldsymbol{R} \cup\{-\infty\})$ auf $H_{\zeta_{*}}^{r}(Q)$ stetig 
fortsetzbar sind. Die Menge dieser auf $H_{\zeta_{*}}^{r}(Q)$ fortgesetzten Opcratoren bczcichncn wir mit $\mathscr{A}_{r}^{t}\left(\zeta_{*}\right)$, und sie bilden $H_{\zeta^{*}}^{r}(Q)$ in $H_{\zeta^{*}}^{r-t}(Q)$ stetig ab. Dieses Ergtbnis läßt sich auch auf negative $r$ erweitern.

5. Die Räume $H_{\zeta_{*}}^{r}(Q)$ erweisen sich nun als angemsccen, um in diesen die Existenz periodischer Lösungen der Gleichung (0.7) mit Operatoren aus $\mathscr{A}_{r}^{t}\left(\zeta_{*}\right)$ $\mathrm{zu}$ untersuchen.

Einen Operator $A(D): S \rightarrow S$ aus der Klasse $\mathscr{A}^{t}\left(\zeta_{*}\right)$ nennen wir $\zeta_{*}$-elliptisch, falls für die erzeugende Abbildung $A: Z^{n} \rightarrow C$ mit zwei Konstanten $C>0$ und $\varrho \geqq 0$ eine Abschätzung der Form

$$
|A(k)| \geqq C \zeta_{*}^{t}(k) \text { für alle } k \in Z^{n} \text { mit }|k| \geqq \varrho
$$

gilt. Ist der Operator $A(D) \zeta_{*}$-elliptisch, so existiert zu dem auf $H_{\zeta_{*}}^{r}(Q)$ stetig fortgesetzten Operator $A_{r}(D)\left(\epsilon \mathscr{A}_{r}^{t}\left(\zeta_{*}\right)\right)$ ein Operator $B_{r-t}(D) \in \mathscr{A}_{r-t}^{-t}\left(\zeta_{*}\right)$ und ein endlicher Operator $R_{r}(D) \in \mathscr{A}_{r}^{-\infty}\left(\zeta_{*}\right)$ (dessen Bild endlichdimensional ist) derart, daß

$$
B_{r-t}(D) \circ A_{r}(D)=I_{r}-R_{r}(D)
$$

gilt, wobei $I_{r}$ der identische Operator auf $H_{\zeta_{*}}^{r}(Q)$ ist.

6. In Abschnitt 5 werden einige Beispiele angegeben. Zunächst wird gezeigt, daß jeder hypoelliptische Differentialoperator $P(D)$, der von einem Polynom $P$ erzeugt ist, $\zeta_{*}$-elliptisch mit $\zeta_{*}(k)=\left(1+|P(k)|^{2}\right)^{1 / 2}$ ist. Ferner werden wir zeigen, daß jeder $t$-koerzitive Differentialoperator $P(D)$ (man vergleiche [8]) $\zeta_{x}$-elliptisch ist. Schließlich wird noch eine Klasse von Integraloperatoren vom Faltungstyp erwähnt, die in die Menge der betrachteten Operatoren fällt.

7. Jeder endliche Operator ist (für jedes $\zeta_{*}$ ) $\zeta_{*}$-glättend. In Abschnitt 6 wird eine hinreichende Bedingung gegeben, damit ein (nicht notwendigerweise endlicher) Operator $\zeta_{*}$-glättend ist.

In Abschnitt 7 wird der Zusammenhang der Kompaktheit der Einbettung von $H_{\zeta_{*}}^{r}(Q)$ in $L_{\text {per }}^{2}(Q)$ und die Äquivalenz der Existenz einer Parametrix mit $\operatorname{der} \zeta_{*}$-Elliptizität eines Operators aus $\mathscr{A}^{t}\left(\zeta_{*}\right)$ untersucht.

Schließlich wird in Abschnitt 8 für $\zeta_{*}$-elliptische Operatoren eine erweiterte Gårdingsche Ungleichung hergeleitet. In dem Fall, daß die eben erwähnte Einbettung kompakt ist, folgt umgekehrt aus der Gültigkeit dieser Gårdingschen Ungleichung die $\zeta_{*}$-Elliptizität.

Die Autoren möchten an dieser Stelle Herrn I. S. Louhivaara für die Hilfe beim Entstehen dieser Arbeit danken. 


\section{Die Algebra $\mathscr{A}^{\infty}\left(\xi_{*}\right)$. Der symbolische Kalkül}

1.1. Es sei $S$ die Menge der Funktionen $\varphi: \boldsymbol{R}^{n} \rightarrow \boldsymbol{C}$, die sich als endliche Summen der Gestalt

$$
\varphi(x)=(2 \pi)^{-n / 2} \sum_{k \in Z^{n}} \varphi_{k} e^{i(k, x)}, \quad x \in \boldsymbol{R}^{n},
$$

mit Koeffizienten $\varphi_{k} \in \boldsymbol{C}$ darstellen lassen; d.h. zu jedem solchen $\varphi$ sind die Koeffizienten $\varphi_{k}$ gleich Null außer für eine endliche Menge von Elementen $k \in Z^{n}$. Die Elemente aus $S$ sind auf $\boldsymbol{R}^{n}$ beliebig oft differenzierbar und periodisch bezüglich

$$
Q:=\left\{x \mid x=\left(x_{1}, \ldots, x_{n}\right) \in \boldsymbol{R}^{n},-\pi \leqq x_{j}<\pi, j=1, \ldots, n\right\} .
$$

Für eine beliebige Abbildung $\zeta: Z^{n} \rightarrow C$ setzen wir

$$
\zeta_{*}(k):=\left(1+|\zeta(k)|^{2}\right)^{1 / 2} \quad \text { für alle } k \in Z^{n} .
$$

Die Menge der Abbildungen $A: Z^{n} \rightarrow C$, die mit einem festen $t \in \boldsymbol{R}$ und mit $C_{t, A} \in \boldsymbol{R}, C_{t, A}>0$ der Abschätzung

$$
|A(k)| \leqq C_{t . A} \zeta_{*}^{t}(k) \text { für alle } k \in Z^{n}
$$

genügen, bezeichnen wir mit $S^{t}\left(\zeta_{*}\right)$. Unter $S^{\infty}\left(\zeta_{*}\right)$ verstehen wir die Menge

$$
S^{\infty}\left(\zeta_{*}\right)=\bigcup_{t \in R} S^{t}\left(\zeta_{*}\right)
$$

Für ein Element $A \in S^{\infty}\left(\zeta_{*}\right)$ definieren wir die $\zeta_{*}$-Ordnung durch

$$
\operatorname{ord}_{\zeta_{*}} A:=\inf \left\{t \in \boldsymbol{R}|| A(k) \mid \leqq C_{t, A} \zeta_{*}^{t}(k) \text { für alle } k \in Z^{n}\right\} \text {. }
$$

Jedes $A \in S^{\infty}\left(\zeta_{*}\right)$ definiert nun auf $S$ einen Operator

$$
A(D): S \rightarrow S
$$

mit

$$
(A(D) \varphi)_{k}=A(k) \varphi_{k} \quad \text { für alle } k \in Z^{n} .
$$

Der Operator $A(D)$ ist damit wohldefiniert, da die Koeffizienten $\varphi_{k}$ nach Voraussetzung für nur endlich viele Indizes $k \in Z^{n}$ von Null verschieden sind.

Die Menge der Operatoren $A(D)$, die je von einer Abbildung $A \in S^{\infty}\left(\zeta_{*}\right)$ erzeugt werden, deren $\zeta_{*}$-Ordnung kleiner oder gleich $t$ ist, $\operatorname{ord}_{\zeta_{*}} A \leqq t$, bezeichnen wir mit $\mathscr{A}^{t}\left(\zeta_{*}\right)$. Weiterhin setzen wir

$$
\mathscr{A}^{\infty}\left(\zeta_{*}\right)=\bigcup_{t \in R} \mathscr{A}^{t}\left(\zeta_{*}\right)
$$

Für $A \in S^{\infty}\left(\zeta_{*}\right)$ ist der Operator $A(D)$ ein Element aus $\mathscr{A}^{\infty}\left(\zeta_{*}\right)$. Umgekehrt existiert für $A(D) \in \mathscr{A}^{\infty}\left(\zeta_{*}\right)$ ein $t \in \boldsymbol{R}$ mit $A(D) \in \mathscr{A}^{t}\left(\zeta_{*}\right)$, also existiert für jedes vorgegebene $\varepsilon>0$ ein $A \in S^{t+\varepsilon}\left(\zeta_{*}\right)$ derart, daß der von $A$ erzeugte Operat $\_$r $A(D)$ ist. 
Da für $A(D) \in \mathscr{A}^{t}\left(\zeta_{*}\right)$ und $B(D) \in \mathscr{A}^{r}\left(\zeta_{*}\right)$ mit zwei Zahlen $\alpha, \beta \in C$ die Relationen $\alpha A(D) \in \mathscr{A}^{t}\left(\zeta_{*}\right)$ bzw. $\beta B(D) \in \mathscr{A}^{r}\left(\zeta_{*}\right)$ und folglich $\alpha A(D)+\beta B(D) \in \mathscr{A}^{\max \{t, r\}}\left(\zeta_{*}\right)$ gelten, ist $\mathscr{A}^{t}\left(\zeta_{*}\right)(t \leqq \infty)$ ein komplexer linearer Raum. Für jedes Paar $t<r \leqq \infty$ ist $\mathscr{A}^{t}\left(\zeta_{*}\right)$ ein Unterraum von $\mathscr{A}^{r}\left(\zeta_{*}\right)$.

1.2. Wir wollen die Struktur einer Algebra in $\mathscr{A}^{\infty}\left(\zeta_{*}\right)$ einführen. Dafür erklären wir für zwei Operatoren $A(D) \in \mathscr{A}^{t}\left(\zeta_{*}\right)$ und $B(D) \in \mathscr{A}^{r}\left(\zeta_{*}\right)$ die Komposition $T(D)=B(D) \circ A(D): S \rightarrow S$ durch

$$
T(D)=B(D)(A(D) \varphi) \text { für } \varphi \in S .
$$

Lemma 1.1. Die Komposition $T(D)=B(D) \circ A(D)$ wird von der durch

$$
T(k)=B(k) A(k) \quad \text { für } \quad k \in Z^{n}
$$

erklärten Abbildung $T: Z^{n} \rightarrow C$ erzeugt. $T(k) \varphi_{k}$.

Beweis. Für $\varphi \in S$ gilt $(T(D) \varphi)_{k}=(B(D)(A(D) \varphi))_{k}=B(k)(A(D) \varphi)_{k}=B(k) A(k) \varphi_{k}=$

Hiermit wird $\mathscr{A}^{\infty}\left(\zeta_{*}\right)$ eine Algebra, d.h. es gilt

1) $\mathscr{A}^{\infty}\left(\zeta_{*}\right)=\bigcup_{t \in R} \mathscr{A}^{t}\left(\zeta_{*}\right)$

2) $\alpha \mathscr{A}^{t}\left(\zeta_{*}\right)+\beta \mathscr{A}^{t}\left(\zeta_{*}\right) \subset \mathscr{A}^{t}\left(\zeta_{*}\right)$ für alle $t \in \boldsymbol{R}$ und $\alpha, \beta \in \boldsymbol{C}$,

3) $\mathscr{A}^{r}\left(\zeta_{*}\right) \circ \mathscr{A}^{t}\left(\zeta_{*}\right) \subset \mathscr{A}^{r+t}\left(\zeta_{*}\right)$ für alle $r, t \in \boldsymbol{R}$.

Einen Operator $A(D)$ aus $\mathscr{A}^{t}\left(\zeta_{*}\right)$ nennen wir $\zeta_{*}$-elliptisch, wenn zwei Konstanten $\varrho \geqq 0$ und $C>0$ so existieren, da

$$
|A(k)| \geqq C \zeta_{*}^{t}(k) \text { für alle } k \in Z^{n} \quad \text { mit } \quad|k| \geqq \varrho
$$

gilt. Im Falle $\zeta(k)=|k|$ für $k \in Z^{n}$ und also $\zeta_{*}^{t}(k)=\left(1+|k|^{2}\right)^{t / 2}$ fällt diese Bedingung mit der ,,̈̈blichen“ Elliptizitätsforderung zusammen (man vergleiche BersSchechter [1], S. 165, Eells [2], S. 107).

1.3. Einen Operator $R(D)$ aus $\mathscr{A}^{\infty}\left(\zeta_{*}\right)$ mit $\operatorname{ord}_{*} R=-\infty$ nennen wir $\zeta_{*}$ glättend. Die Menge aller $\zeta_{*}$-glättenden Operatoren bezeichnen wir mit $\mathscr{A}^{-\infty}\left(\zeta_{*}\right)$. Offensichtlich gilt

$$
\mathscr{A}^{-\infty}\left(\zeta_{*}\right)=\bigcap_{t \in R} \mathscr{A}^{t}\left(\zeta_{*}\right) .
$$

Einen Operator $R(D) \in \mathscr{A}^{\infty}\left(\zeta_{*}\right)$, für den das Bild $R(D) S$ von $S$ endlichdimensional ist, nennen wir endlich.

Die endlichen Operatoren sind genau die Operatoren $R(D)$, die je von einer Abbildung $R: Z^{n} \rightarrow C$ mit der Eigenschaft, da $R(k) \neq 0$ nur für endlich viele $k \in Z^{n}$ ist, erzeugt werden.

Jeder endliche Operator ist (für jedes $\zeta_{*}$ ) $\zeta_{*}$-glättend: Denn mit $E=$ $\left\{k \in Z^{n} \mid R(k) \neq 0\right\}$ erkläre man für $t \in \boldsymbol{R}$ die Konstante

$$
C_{t, R, \zeta_{*}}=\frac{\max _{k \in E}|R(k)|}{\min _{k \in E} \zeta_{*}^{t}(k)} .
$$


Dann gilt die Abschätzung

mit jedem $t \in \boldsymbol{R}$.

$$
|R(k)| \leqq C_{t, R, \zeta_{*}} \zeta_{*}^{t}(k) \quad \text { für alle } k \in Z^{n},
$$

Umgekehrt braucht ein $\zeta_{*}$-glättender Operator $R(D)$ nicht endlich sein. Als Beispiel dafür nehme man im Falle eines bestimmten $\zeta_{*}$ den durch die Abbildung $R: Z^{n} \rightarrow C$ mit $R(k):=\exp \left(-\left|\zeta_{*}(k)\right|\right)$ erklärten Operator.

Falls zu einem Operator $A(D) \in \mathscr{A}^{t}\left(\zeta_{*}\right)(t \in R)$ ein Operator $B(D) \in \mathscr{A}^{-t}\left(\zeta_{*}\right)$ mit erzeugender Funktion $B \in S^{-t}\left(\zeta_{*}\right)$ und ein $\zeta_{*}$-glättender Operator $R(D) \in \mathscr{A}^{-\infty}\left(\zeta_{*}\right)$ derart existieren, daß

$$
B(D) \circ A(D)=I-R(D)
$$

gilt, wobei $I$ den identischen Operator auf $S$ bezeichnet, nennen wir $B(D)$ eine $\zeta_{*}$-Parametrix von $A(D)$ (man vergleiche [9], S. 60; [10], S. 41).

\subsection{Es gilt nun}

Satz 1.2. Ein Operator $A(D) \in \mathscr{A}^{t}\left(\zeta_{*}\right)(t \in \boldsymbol{R})$ ist dann und nur dann $\zeta_{*}$-elliptisch, wenn eine $\zeta_{*}$-Parametrix $B(D) \in \mathscr{A}^{-t}\left(\zeta_{*}\right)$ derart existiert, daß der in (1.8) vorkommende Operator $R(D)$ endlich ist.

Beweis. A. Sei zuerst $A(D) \zeta_{*}$-elliptisch. Dann wird $N^{\prime}=\left\{k \in Z^{n} \mid A(k)=0\right\}$ aufgrund von (1.7) endlich sein, und folglich existiert eine solche Konstante $C^{\prime}$, daß

$$
\frac{1}{|A(k)|} \leqq C^{\prime} \frac{1}{\zeta_{*}^{t}(k)} \quad \text { für alle } \quad k \in N:=Z^{n}-N^{\prime}
$$

gilt. Die durch die Vorschrift

$$
B(k)=\left\{\begin{array}{cc}
0, & k \in N^{\prime}, \\
\frac{1}{A(k)}, & k \in N,
\end{array}\right.
$$

definierte Abbildung $B: Z^{n} \rightarrow C$ erfüllt die Ungleichung

$$
|B(k)| \leqq C^{\prime} \frac{1}{\zeta_{*}^{t}(k)} \quad \text { für alle } \quad k \in Z^{n},
$$

und der entsprechende Operator $B(D): S \rightarrow S$ ist ein Element in $\mathscr{A}^{-t}\left(\zeta_{*}\right)$. Andererseits gehört die durch die Vorschrift

$$
R(k)= \begin{cases}1, & k \in N^{\prime}, \\ 0, & k \in N,\end{cases}
$$

erklärte Abbildung $R: Z^{n} \rightarrow C$ zu $S^{-\infty}\left(\zeta_{*}\right)$ und erzeugt somit einen endlichen Operator $R(D): S \rightarrow S$, für den bei beliebiger Wahl von $\varphi \in S$

$$
(R(D) \varphi)_{k}= \begin{cases}\varphi_{k}, & k \in N^{\prime}, \\ 0, & k \in N,\end{cases}
$$


gilt. Somit hat man

$$
(B(D) \circ A(D) \varphi)_{k}=B(k) A(k) \varphi_{k}= \begin{cases}0, & k \in N^{\prime} \\ \varphi_{k}, & k \in N .\end{cases}
$$

Folglich gilt $B(D) \circ A(D)=I-R(D)$, und $B(D)$ ist die erwünschte $\zeta_{*}$-Parametrix.

B. $\mathrm{Zu} A(D) \in \mathscr{A}^{t}\left(\zeta_{*}\right)$ sei $B(D) \in \mathscr{A}^{-t}\left(\zeta_{*}\right)$ eine $\zeta_{*}$-Parametrix, und weiter gelte

$$
B(D) \circ A(D)=I-R(D)
$$

mit einem endlichen Operator $R(D)$. Somit existiert eine Abbildung $R: Z^{n} \rightarrow C$ und eine Konstante $\varrho>0$ so, daß

gilt. Also folgt

$$
R(k)=0 \text { für alle } \quad k \in Z^{n} \text { mit }|k|>\varrho
$$

$$
B(k) A(k)=1 \text { für alle } k \in Z^{n} \quad \text { mit } \quad|k|>\varrho .
$$

Da $B \in S^{-t}\left(\zeta_{*}\right)$, hat man nach (1.3)

$$
|A(k)|=\frac{1}{|B(k)|} \geqq \frac{1}{C_{t, B}} \zeta_{*}^{t}(k) \quad \text { für } \quad|k|>\varrho,
$$

und der Operator $A(D)$ ist $\zeta_{*}$-elliptisch.

\section{Die Räume $H_{\zeta_{*}}^{r}(Q)$ für $r \in \boldsymbol{R}$}

2.1. Der Fall $r=0$. Es sei wie in $1.1 Q$ der Periodenwürfel in $\boldsymbol{R}^{n}$. Die Menge der auf $\boldsymbol{R}^{n}$ definierten beliebig oft differenzierbaren Funktionen, die bezüglich $Q$ periodisch sind, bezeichnen wir mit $C_{\text {per }}^{\infty}(Q)$. Mit $L_{\text {per }}^{2}(Q)$ bezeichnen wir die Menge aller bezüglich $Q$ periodischen komplexwertigen Funktionen, die auf dem Periodenwürfel meßbar und quadratintegrierbar sind. Für $f, g \in L_{\text {per }}^{2}(Q)$ schreiben wir

und $\|f\|_{0}:=(f, f)_{0}^{1 / 2}$. Die Menge

$$
(f, g)_{0}:=\int_{Q} \overline{f(x)} g(x) d x
$$

$$
\left\{(2 \pi)^{-n / 2} e^{i(k, \cdot)} \mid k \in Z^{n}\right\}
$$

bildet bezüglich obigen Skalarproduktes eine Orthonormalbasis in $L_{\mathrm{per}}^{2}(Q)$. Folglich liegt die Menge $S$ aus 1.1 dicht in $L_{\text {per }}^{2}(Q)$ und jedes Element $f \in L_{\text {per }}^{2}(Q)$ hat die Darstellung

$$
f(x)=(2 \pi)^{-n / 2} \sum_{k \in \mathbb{Z}^{n}} f_{k} e^{i(k, x)}, \quad x \in \boldsymbol{R}^{n},
$$

wobei für den Fourierkoeffizienten $f_{k}, k \in Z^{n}$,

gilt.

$$
f_{k}=(2 \pi)^{-n / 2} \int_{Q} f(x) e^{-i(k, x)} d x
$$


2.2. Der Fall $r>0$. Für eine beliebige Abbildung $\zeta: Z^{n} \rightarrow C$ erklären wir wieder $\zeta_{*}$ gemäß (1.2). Auf $S$ definieren wir nun für jedes reelle $r \geqq 0$ durch

$$
(\varphi, \psi)_{r, \zeta_{*}}:=\sum_{k \in Z^{n}} \zeta_{*}^{2 r}(k) \bar{\varphi}_{k} \psi_{k}
$$

ein Skalarprodukt mit entsprechender Norm $\|\varphi\|_{r, \zeta_{*}}$.

Man bemerke, daß $(\varphi, \psi)_{0, \zeta_{*}}=(\varphi, \psi)_{0}$ gilt.

Durch Vervollständigen von $S$ bezüglich des Skalarproduktes $(\varphi, \psi)_{r, \zeta_{*}}$ entsteht der Hilbertraum $H_{\zeta_{*}}^{r}(Q)$. $\mathrm{Zu}$ jedem Element $u \in H_{\zeta_{*}}^{r}(Q)$ existiert somit eine Folge $\left\{\varphi_{v}(\cdot)\right\}_{v \in N}$ von Elementen $\varphi_{v} \in S$, mit $\left\|u-\varphi_{v}\right\|_{r, \zeta_{*}} \rightarrow 0$ für $v \rightarrow \infty$.

Lemma 2.1. A. Für $0 \leqq s<r$ ist die Einbettung von $H_{\zeta_{*}}^{r}(Q)$ in $H_{\zeta_{*}}^{s}(Q)$ stetig: $H_{\zeta_{*}}^{r}(Q) \subset H_{\zeta_{*}}^{s}(Q)$. Insbesondere gilt $H_{\zeta_{*}}^{r}(Q) \subset H_{\zeta_{*}}^{0}(Q)=L_{\text {per }}^{2}(Q)$ für alle $r>0$.

B. Gilt für zwei Abbildungen $\zeta_{1}$ und $\zeta_{2}$ für alle $k \in Z^{n}$ mit $|k| \geqq \varrho, \varrho \geqq 0$, $\zeta_{1}(k) \leqq \zeta_{2}(k)$, so ist $H_{\zeta_{2 *}}^{r}(Q)$ stetig in $H_{\zeta_{1 *}}^{r}(Q)$ für jedes $r>0$ eingebettet.

C. Für jedes $u \in H_{\zeta_{*}}^{r}(Q)$ ist durch $\left\{\zeta_{*}^{r}(k) u_{k}\right\}_{k \in Z^{n}}$ ein Element in $\ell^{2}$, in dem Raum der quadratisch summierbaren Folgen in $C$, erklärt. Ist umgekehrt $\left\{\zeta_{*}^{r}(k) u_{k}\right\}_{k \in Z^{n}}$ eine Folge in $\ell^{2}$, so ist durch

$$
u(\cdot)=(2 \pi)^{-n / 2} \sum_{k \in Z^{n}} u_{k} e^{i(k, \cdot)}
$$

ein Element in $H_{\zeta_{*}}^{r}(Q)$ definiert.

2.3. Der Fall $r<0$. Es gilt zunächst

Lemma 2.2. Für alle $f \in L_{\mathrm{per}}^{2}(Q)=H_{\zeta_{*}}^{0}(Q)$ und alle $u \in H_{\zeta_{*}}^{r}(Q)$ mit $r \geqq 0$ gilt

$$
\left|(f, u)_{0}\right| \leqq\|f\|_{0}\|u\|_{r, \zeta_{*}} .
$$

Folglich wird für jedes $f \in L_{\text {per }}^{2}(Q)$ ein lineares und stetiges Funktional $l_{f}: H_{\zeta_{*}}^{r}(Q) \rightarrow C$ durch $l_{f}(u):=(f, u)_{0}$ erklärt. Die Menge dieser Funktionale bezeichnen wir mit $\mathscr{L}$. Definieren wir für ein $f \in L_{\text {per }}^{2}(Q)$ die Norm

$$
\|f\|_{-r, \zeta_{*}}:=\left\|\mid l_{f}\right\|_{-r, \zeta_{*}}=\sup _{u \in H_{\zeta_{*}}^{r}(Q)} \frac{\left|(f, u)_{0}\right|}{\|u\|_{r, \zeta_{*}}}
$$

und bezeichnen wir den (topologischen) Dualraum von $H_{\zeta_{*}}^{r}(Q)$ mit $\left(H_{\zeta_{*}}^{r}(Q)\right)^{\prime}$, so gilt

Lemma 2.3. Für jedes reelle $r \geqq 0$ liegt $\mathscr{L}$ bezüglich der Norm \|\|\|\|$_{-r, \zeta_{*}}$ dicht in $\left(H_{\zeta_{*}}^{r}(Q)\right)^{\prime}$.

Beweis. Für $r=0$ ist die Behauptung trivial. Sei $r>0$. Wenn nun $\mathscr{L}$ nicht dicht in $\left(H_{\zeta_{*}}^{r}(Q)\right)^{\prime}$ liegt, so existiert ein Funktional $T \neq 0$ aus dem Bidualraum von $H_{\zeta_{*}}^{r}(Q)$ mit Kern $T \supset \mathscr{L}$. Somit existiert ein eindeutig bestimmtes Element 
$u \in H_{\zeta_{*}}^{r}(Q)$, mit dem $T\left(l_{f}\right)=(f, u)_{0}$. für alle $l_{f} \in \mathscr{L}$ gilt. Wegen Kern $T \supset \mathscr{L}$ folgt $(f, u)_{0}=0$ für alle $f \in L_{\text {per }}^{2}(Q)$ und somit $u=0$, was im Widerspruch zu $T \neq 0$ steht.

Somit gilt

Lemma 2.4. Der Raum $\left(H_{\zeta_{*}}^{r}(Q)\right)^{\prime}$ ist isomorph zur Abschließung von $L_{\mathrm{per}}^{2}(Q)=$ $H_{\zeta_{*}}^{0}(Q)$ bezüglich der Norm $\|\cdot\|_{-r, \zeta_{*}}$.

Für den Abschluß $H_{\zeta_{*}}^{-r}(Q)(r>0)$ von $L_{\text {per }}^{2}(Q)$ bezüglich der Norm $\|\cdot\|_{-r, \zeta_{*}}$ gilt somit

$$
\left(H_{\zeta_{*}}^{r}(Q)\right)^{\prime}=H_{\zeta_{*}^{-r}}^{-r}(Q) .
$$

Wir bemerken, daß im Falle $\zeta_{*}(k)=\left(1+|k|^{2}\right)^{1 / 2}$ der Raum $H_{\zeta_{*}}^{t}(Q)$ mit dem Raum $H_{t}$ aus Bers-Schechter [1], S. 167, zusammenfällt. Man vergleiche auch Louhivaara-Simader [8], S. 88.

2.4. Um die später benötigte stetige Fortsetzbarkeit eines Operators $A(D): S \rightarrow S$ aus $\mathscr{A}^{t}\left(\zeta_{*}\right)$ auf einen Raum $H_{\zeta_{*}}^{r}(Q)(t, r \in \boldsymbol{R})$ zeigen zu können, werden wir eine der Formel (2.1) analoge Darstellung für die negative Norm herleiten: Dazu erklären wir zuerst die $r$-te Potenz des Operators $\zeta_{*}(D)$ für beliebiges $r \in \boldsymbol{R}$,

durch

$$
\zeta_{*}^{r}(D): S \rightarrow S,
$$

$$
\left(\zeta_{*}^{r}(D) \varphi\right)_{k}:=\zeta_{*}^{r}(k) \varphi_{k} \text { für alle } k \in Z^{n} .
$$

Im Falle $r=0$ fällt dieser Operator mit dem identischen Operator des Raumes $S$ zusammen.

Ist $r>0$, so gilt gemäß (2.1) und (2.3)

$$
\|\varphi\|_{r, \zeta_{*}}=\left\|\zeta_{*}^{r}(D) \varphi\right\|_{0}
$$

für alle $\varphi \in S$. Für negative $r$ zeigen wir:

Lemma 2.5. Mit $r \in \boldsymbol{R}, r>0$ gilt

$$
\|\varphi\|_{-r, \zeta_{*}}=\left\|\zeta_{*}^{-r}(D) \varphi\right\|_{0} \text { für alle } \varphi \in S .
$$

Beweis. Für $\varphi, \psi \in S, \psi \neq \equiv$ gilt, weil $\zeta_{*}^{r}(k) \neq 0$ für alle $k \in Z^{n}$ ist,

Daraus folgt nach (2.4)

$$
(\varphi, \psi)_{0}=\left(\zeta_{*}^{-r}(D) \varphi, \zeta_{*}^{r}(D) \psi\right)_{0} .
$$

$$
\frac{\left|(\varphi, \psi)_{0}\right|}{\|\psi\|_{r, \zeta_{*}}}=\frac{\left|\left(\zeta_{*}^{-r}(D) \varphi, \zeta_{*}^{r}(D) \psi\right)_{0}\right|}{\left\|\zeta_{*}^{r}(D) \psi\right\|_{0}}
$$

Wegen $\zeta_{*}^{r}(k) \neq 0$ gibt es zu jedem $\psi \in S$ mit Fourierkoeffizienten $\psi_{k}$ immer ein eindeutig bestimmtes $\chi \in S$ mit Fourierkoeffizienten $\chi_{k}=\zeta_{*}^{r}(k) \psi_{k}$. $\left(\zeta_{*}^{r}(D)\right.$ ist ein bijektiver Operator von $S$ auf $S$.) Aufgrund der Dichtheit von $S$ in $H_{\zeta_{*}}^{r}(Q)$ 
gilt gemäß (2.6)

und

$$
\sup _{\psi \in S, \psi \neq 0} \frac{\left|(\varphi, \psi)_{0}\right|}{\|\psi\|_{r, \zeta_{*}}}=\sup _{\substack{\chi=\zeta_{*}^{-r}(D) \psi, \psi \in S, \psi \neq 0}} \frac{\left|\left(\zeta_{*}^{-r}(D) \varphi, \chi\right)_{0}\right|}{\|\chi\|_{0}},
$$

$$
\|\varphi\|_{-r, \zeta^{*}}=\left\|\zeta_{*}^{-r}(D) \varphi\right\|_{0}
$$

\section{Fortsetzung der Operatoren aus $\mathscr{A}^{\infty}\left(\zeta_{*}\right)$ auf die Räume $H_{\zeta^{*}}^{r}(Q)$}

3.1. Bisher haben wir jeder Abbildung $A \in S^{t}\left(\zeta_{*}\right)$ einen Operator $A(D): S \rightarrow S$ aus $\mathscr{A}^{t}\left(\zeta_{*}\right)$ zugeordnet. Da für jedes $r \in \boldsymbol{R}$ die Menge $S$ dicht in $H_{\zeta_{*}}^{r}(Q)$ liegt, werden wir nun den Operator $A(D)$ auf $H_{\zeta_{*}}^{r}(Q)$ fortsetzen. Als Vorbereitung dafür geben wir

Lemma 3.1. Seien $r, t \in \boldsymbol{R}$. Für den von $A \in S^{t}\left(\zeta_{*}\right)$ erzeugten Operator $A(D): S \rightarrow S$ gilt mit einer Konstanten $C_{t, A}$

$$
\|A(D) \varphi\|_{r-t, \zeta_{*}} \leqq C_{t, A}\|\varphi\|_{r, \zeta_{*}} \text { für alle } \varphi \in S .
$$

Beweis. Aufgrund von (1.3) gilt für jedes $\varphi \in S$

$$
\begin{gathered}
\|A(D) \varphi\|_{r-t, \zeta_{*}}^{2}=\sum_{k \in Z^{n}}|A(k)|^{2}\left|\varphi_{k}\right|^{2} \zeta_{*}^{2(r-t)}(k) \\
\leqq C_{t, A}^{2} \sum_{k \in Z^{n}}\left|\varphi_{k}\right|^{2} \zeta_{*}^{2 r}(k)=C_{t, A}^{2}\|\varphi\|_{r, \zeta_{*}}^{2} .
\end{gathered}
$$

Nach dem eben bewiesenen Lemma läßt sich der Operator $A(D)$ aus $\mathscr{A}^{t}\left(\zeta_{*}\right)$ für jedes $r \in \boldsymbol{R}$ stetig zu einem stetigen Operator

$$
A_{r}(D): H_{\zeta_{*}}^{r}(Q) \rightarrow H_{\zeta_{*}}^{r-t}(Q)
$$

fortsetzen.

Die Menge aller Operatoren, die durch stetige Fortsetzung der Operatoren aus $\mathscr{A}^{t}\left(\zeta_{*}\right), t \in \boldsymbol{R} \cup\{-\infty, \infty\}$ auf $H_{\zeta_{*}}^{r}(Q)$ entstehen, bezeichnen wir mit $\mathscr{A}_{r}^{t}\left(\zeta_{*}\right)$. Die Operatoren der Menge $\mathscr{A}_{r}^{-\infty}\left(\zeta_{*}\right)$ nennen wir $\zeta_{*}$-glättend in $H_{\zeta_{*}}^{r}(Q)$.

3.2. Für $A \in S^{t}\left(\zeta_{*}\right)$ und $B \in S^{S}\left(\zeta_{*}\right)$ ist nach Lemma 1.1 die Komposition $T(D)=B(D) \circ A(D): S \rightarrow S$ der Operatoren $A(D) \in \mathscr{A}^{t}\left(\zeta_{*}\right)$ und $B(D) \in \mathscr{A}^{s}\left(\zeta_{*}\right)$ von der durch $T(k)=B(k) A(k)$ erklärten Abbildung $T: Z^{n} \rightarrow C$ aus $S^{t+s}\left(\zeta_{*}\right)$ erzeugt.

Lemma 3.2. Die Komposition $T(D) \in \mathscr{A}^{t+s}\left(\zeta_{*}\right)$ der Operatoren $A(D) \in \mathscr{A}^{t}\left(\zeta_{*}\right)$ und $B(D) \in \mathscr{A}^{s}\left(\zeta_{*}\right)$ mit erzeugenden Funktionen $A \in S^{t}\left(\zeta_{*}\right)$ und $B \in S^{s}\left(\zeta_{*}\right)$ genügt für jedes $r \in \boldsymbol{R}$ der Ungleichung

$$
\|T(D) \varphi\|_{r-t-s} \leqq C_{s, t, A, B}\|\varphi\|_{r} \text { für alle } \varphi \in S .
$$


Beweis. Weil nach Lemma 1.1 für $\varphi \in S$

gilt, ergibt sich

$$
(T(D) \varphi)_{k}=T(k) \varphi_{k}=B(k) A(k) \varphi_{k}
$$

$$
\begin{aligned}
\|T(D) \varphi\|_{r-t-s}^{2}= & \sum_{k \in Z^{n}}\left|(T(D) \varphi)_{k}\right|^{2} \zeta_{*}^{2(r-t-s)}(k)=\sum_{k \in Z^{n}}|B(k)|^{2}|A(k)|^{2}\left|\varphi_{k}\right|^{2} \zeta_{*}^{2(r-t-s)}(k) \\
& \leqq C_{s, B}^{2} C_{t, A}^{2} \sum_{k \in Z^{n}}\left|\varphi_{k}\right|^{2} \zeta_{*}^{2 r}(k) \leqq C_{s, t, A, B}^{2}\|\varphi\|_{r, \zeta_{*}}^{2} .
\end{aligned}
$$

Also läßt sich der Operator $T(D)$ für jedes $r \in \boldsymbol{R}$ stetig zu einem (stetigen) Operator $T_{r}(D): H_{\zeta_{*}}^{r}(Q) \rightarrow H_{\zeta_{*}}^{r-t-s}(Q)$ fortsetzen.

Andererseits kann man die Komposition der beiden Hilbertraumoperatoren $A_{r}(D): H_{\zeta_{*}}^{r}(Q) \rightarrow H_{\zeta_{*}}^{r-t}(Q) \quad$ und $\quad B_{r-t}(D): H_{\zeta_{*}}^{r-t}(Q) \rightarrow H_{\zeta_{*}}^{r-t-s}(Q)$ betrachten, und es gilt (wegen der Dichtheit von $S$ in allen Räumen $\left.H_{\zeta_{*}}^{l}(Q), l \in R\right) T_{r}(D)=$ $B_{r-t}(D) \circ A_{r}(D)$ für jedes $r \in \boldsymbol{R}$.

Anstelle der Eigenschaft 3) der Algebra $\mathscr{A}^{\infty}\left(\zeta_{*}\right)$ haben wir jetzt

$$
\mathscr{A}_{r-t}^{s}\left(\zeta_{*}\right) \circ \mathscr{A}_{r}^{t}\left(\zeta_{*}\right) \subset \mathscr{A}_{r-s-t}^{s+t}\left(\zeta_{*}\right)
$$

\section{Periodische Lösungen von Operatorgleichungen auf $H_{\zeta_{*}}^{r}(Q)$}

4.1. Wir sind nun in der Lage, die periodischen Lösungen einer Operatorgleichung aufzusuchon.

Problem 4.1. Es seien $A_{r}(D) \in \mathscr{A}_{r}^{t}\left(\zeta_{*}\right)$ und $f \in H_{\zeta_{*}}^{r-t}(Q)$. Gesucht wird die Gesamtheit der Elemente $u \in H_{\zeta_{*}}^{r}(Q)$ für die

$$
A_{r}(D) u=f
$$

gilt.

Man beachte, daß aufgrund der Konstruktion des Operators $A_{r}(D)$ dieses Problem der Aufgabe äquivalent ist: $\mathrm{Zu}$ einem vorgegebenen $f \in H_{\zeta_{*}}^{r-t}(Q)$ mit Fourierkoeffizienten $f_{k}$ bestimme man die Gesamtheit aller solcher Elemente $u \in H_{\zeta_{*}}^{r}(Q)$ mit Fourierkoeffizienten $u_{k}$, daß

$$
A(k) u_{k}=f_{k} \quad \text { für alle } \quad k \in Z^{n}
$$

gilt.

Im Spezialfall, wo $\zeta_{*}(k)=\left(1+|k|^{2}\right)^{1 / 2}$ und $A \in S^{t}\left(\zeta_{*}\right)$ ein Polynom mit konstanten Koeffizienten ist, handelt es sich darum, periodische Lösungen von Differentialgleichungen im Raum $H_{r}$ zu suchen (man vergleiche Bers-Schechter [1], S. 167).

4.2. Ist nun $A(D)$ ein $\zeta_{*}$-elliptischer Operator aus $\mathscr{A}^{t}\left(\zeta_{*}\right)$, so werden wir die Gültigkeit der Fredholmschen Alternative für Problem 4.1 mit dem auf $H_{\zeta_{*}}^{r}(Q)$ fortgesetzten Operator $A_{r}(D)$ zeigen. 
Wegen der $\zeta_{*}$-Elliptizität von $A(D)$ ist die Menge $N^{\prime}:=\left\{k \in Z^{n} \mid A(k)=0\right\}$ endlich. Die bezüglich der Norm $\|\cdot\|_{r-t, \zeta_{*}}$ abgeschlossene lineare Hülle der Menge $\quad\left\{(2 \pi)^{-n / 2} e^{i(k \cdot \cdot)} \mid k \in N, N:=Z^{n}-N^{\prime}\right\} \quad(\subset S)$ bezeichne man mit $\mathscr{N}_{r-t}$. Mit $\mathscr{N}^{\prime}$ bezeichnen wir den $\epsilon$ ndlichdimensionalen linearen Unterraum von $S$, der von der Menge $\left\{(2 \pi)^{-n / 2} e^{i(k, \cdot)} \mid k \in N^{\prime}\right\}$ aufgespannt ist. $\mathscr{N}^{\prime}$ ist dann in jedem $H_{\zeta_{*}}^{r}(Q)$ abgeschlossen.

Satz 4.2. Für einen $\zeta_{*}$-elliptischen Operator $A(D)$ aus $\mathscr{A}^{t}\left(\zeta_{*}\right)$ gilt für Problem 4.1 die Fredholmsche Alternative: Dieses Problem hat eine Lösung dann und nur dann, wenn $f \in \mathscr{N}_{r-t}$ ist; die Lösung ist dann bis auf ein beliebiges additives Element aus $\mathscr{N}^{\prime}$ eindeutig bestimmt.

Beweis. Wir unterscheiden zwei Fälle. A. Ist zunächst $\mathscr{N}^{\prime}=\emptyset$, d.h. $A(k) \neq 0$ für alle $k \in Z^{n}$, so wird für jedes $f \in H_{\zeta_{*}}^{r-t}(Q)$ mit den Fourierkoeffizienten $f_{k}$ ein Element $u \in H_{\zeta_{*}}^{r}(Q)$ mit den Fourierkoeffizienten

$$
u_{k}=\frac{f_{k}}{A(k)} \quad \text { für } \quad k \in Z^{n}
$$

definiert. Wegen der $\zeta_{*}$-Elliptizität (1.7) von $A(D)$ gilt nämlich mit einer passenden Konstanten $C^{\prime}$

und somit

$$
\frac{1}{|A(k)|} \leqq C^{\prime} \frac{1}{\zeta_{*}^{t}(k)} \quad \text { für alle } \quad k \in Z^{n}
$$

$$
\begin{gathered}
\|u\|_{r, \zeta_{*}}^{2}=\sum_{k \in Z^{n}}\left|u_{k}\right|^{2} \zeta_{*}^{2 r}(k)=\sum_{k \in Z^{n}} \frac{\left|f_{k}\right|^{2}}{|A(k)|^{2}} \zeta_{*}^{2 r}(k) \\
\leqq C^{\prime 2} \sum_{k \in Z^{n}}\left|f_{k}\right|^{2} \zeta_{*}^{2(r-t)}(k) \leqq C^{\prime 2}\|f\|_{r-t, \zeta_{*}}^{2} .
\end{gathered}
$$

Offenbar ist das konstruierte Element $u \in H_{\zeta_{*}}^{r}(Q)$ eine Lösung des Problems. In diesem Fall ist $\mathscr{N}_{r-t}=H_{\zeta_{*}}^{r-t}(Q)$ und die Lösung nach (4.3) eindeutig bestimmt $\left(\mathcal{N}^{\prime}=\emptyset\right)$.

B. Ist nun $\mathscr{N}^{\prime} \neq \emptyset$ und existiert zu einem $f \in H_{\zeta_{*}}^{r-t}(Q)$ eine Lösung $u \in H_{\zeta_{*}}^{r}(Q)$ der Gleichung (4.1), so gilt

$$
A(k) u_{k}=f_{k} \text { für alle } k \in Z^{n} .
$$

Hieraus folgt aber $f_{k}=0$ für $k \in N^{\prime}$, und daher $f \in \mathscr{N}_{r-t}$. Ist umgekehrt $f \in \mathscr{N}_{r-t}$, so wird wie im Fall A durch

$$
u_{k}=\frac{f_{k}}{A(k)} \quad \text { für } \quad k \in N
$$

ein Element $u \in H_{\zeta_{*}}^{r}(Q)$ definiert, das eine Lösung der Gleichung (4.1) ist.

Für $g \in \mathscr{N}^{\prime}$ gilt $g_{k}=0$ für $k \in N$ und weiter wegen (4.5) bzw. (4.6)

$$
(A(D)(u+g))_{k}=A(k)\left(u_{k}+g_{k}\right)=f_{k} \text { für alle } k \in Z^{n} .
$$


Aus $A(D) u=f$ und $A(D) v=f$ folgt $(A(D)(u-v))_{k}=0$ und damit

$$
0=(A(D)(u-v))_{k}=A(k)\left(u_{k}-v_{k}\right) \text { für alle } k \in Z^{n} .
$$

Also gilt $u_{k}-v_{k}=0$ für $k \in N$ und somit $u-v \in \mathscr{N}^{\prime}$.

4.3. Jetzt können wir Satz 4.2 mit Hilfe des Begriffes der $\zeta_{*}$-Parametrix unter Berücksichtigung von Satz 1.2 wie folgt neu formulieren:

Satz 4.3. Es sei $A(D)$ ein $\zeta_{*}$-elliptischer Operator aus $\mathscr{A}^{t}\left(\zeta_{*}\right)(t \in \boldsymbol{R})$, und man nehme $r \in \boldsymbol{R}$. Zu dem auf $H_{\zeta_{*}}^{r}(Q)$ fortgesetzten Operator $A_{r}(D) \in \mathscr{A}_{r}^{t}\left(\zeta_{*}\right)$ existiert ein Operator $B_{r-1}(D) \in \mathscr{A}_{r-t}^{-t}\left(\zeta_{*}\right)$ und ein endlicher Operator ${ }^{1} R_{r}(D) \in \mathscr{A}_{r}^{-\infty}\left(\zeta_{*}\right)$ derart, daß

$$
B_{r-t}(D) \circ A_{r}(D)=I_{r}-R_{r}(D)
$$

gilt, wobei $I_{r}$ der identische Operator von $H_{\zeta_{*}}^{r}(Q)$ ist.

Beweis. Nach dem in 3.2 über die Fortsetzung der Komposition Gesagten, setzt man nun den Operator $B(D) \in \mathscr{A}^{-t}\left(\zeta_{*}\right)$ und den endlichen Operator $R(D) \epsilon$ $\mathscr{A}^{-\infty}\left(\zeta_{*}\right)$ aus dem Beweis von Satz 1.2 auf die entsprechenden Räume $H_{\zeta_{*}}^{r-t}(Q)$ bzw. $H_{\zeta_{*}}^{r}(Q)$ fort. (Für den endlichen Operator $R(D)$ bleibt die Wertemenge bei der Fortsetzung auf $H_{\zeta_{*}}^{r}(Q)$ unverändert.)

\section{Beispiele}

5.1. Jeder lineare partielle Differentialoperator $P(D): S \rightarrow S$ mit konstanten Koeffizienten gehört trivialerweise zu der Klasse $\mathscr{A}^{1}\left(\zeta_{*}\right)$ mit $\zeta_{*}(k):=\left(1+|P(k)|^{2}\right)^{1 / 2}$. Im Folgenden werden wir durch einige Beispiele die Klasse der $\zeta_{*}$-elliptischen Operatoren aufhellen.

5.2. Zuerst betrachten wir hypoelliptische Differentialoperatoren. Damit der einem Polynom $P(\xi)$ mit konstanten komplexen Koeffizienten zugeordnete Differentialoperator $P(D)$ hypoelliptisch ist, ist notwendig und hinreichend, daß

$$
\lim _{|\xi| \rightarrow \infty} \frac{\left|P^{(\alpha)}(\xi)\right|}{1+|P(\xi)|}=0
$$

für alle $\alpha \in N_{0}^{n}, \alpha \neq(0, \ldots, 0)$ gilt. (Man vergleiche dazu Hörmander [4], S. 99.)

Der Grad des Polynoms $P$ sei $m$. Aus (5.1) folgt für $|\alpha|=m$

$$
\lim _{|\xi| \rightarrow \infty} \frac{1}{1+|P(\xi)|}=0
$$

${ }^{1}$ Auch in $H_{\zeta_{*}}^{r}(Q)$ heißt ein Operator endlich, falls das Bild von $H_{\zeta_{*}}^{r}(Q)$ unter diesem Operator endlichdimensional ist. 
bzw. $\lim _{|\xi| \rightarrow \infty}|P(\xi)|=\infty$. Somit existiert eine Konstante $\varrho \geqq 0$ derart, daß gilt

$$
|P(\xi)| \geqq 1
$$

für alle $\xi \in \boldsymbol{R}^{n}$ mit $|\xi| \geqq \varrho$. Ferner folgt für $|\xi| \geqq \varrho$

und somit

$$
4|P(\xi)|^{2} \geqq 1+2|P(\xi)|+|P(\xi)|^{2}
$$

$$
|P(\xi)| \geqq \frac{1}{2} \zeta_{*}(\xi) \text { für alle } \xi \in \boldsymbol{R}^{n} \quad \text { mit } \quad|\xi| \geqq \varrho .
$$

Jeder hypoelliptische Differentialoperator $P(D): S \rightarrow S$ mit konstanten Koeffizienten ist also $\zeta_{*}$-elliptisch mit $\zeta_{*}(k):=\left(1+|P(k)|^{2}\right)^{1 / 2}$.

Aufgrund von Lemma 3.1 ist der Operator $P_{r}(D): H_{\zeta_{*}}^{r}(Q) \rightarrow H_{\zeta_{*}}^{r-1}(Q)$ für jedes $r \in \boldsymbol{R}$ stetig. Wegen der $\zeta_{*}$-Elliptizität des Operators $P(D)$ ist die Fredholmsche Alternative (Satz 4.2) anwendbar. Nach Satz 4.3 folgt für jeden hypoelliptischen Operator $P_{r}(D)$ die Existenz einer Parametrix aus $\mathscr{A}_{r-1}^{-1}\left(\zeta_{*}\right)$.

5.3. Nun werden wir zeigen, daß jeder $t$-koerzitive $(t \in \boldsymbol{R}, t>0)$ Differentialoperator $P(D): S \rightarrow S$ mit konstanten Koeffizienten $\zeta_{*}$-elliptisch (mit $\zeta_{*}(k):=$ $\left.\left(1+|P(k)|^{2}\right)^{\mathbf{i} / 2}\right)$ ist.

Ein Operator $P(D)$ heißt $t$-koerzitiv $(t \in \boldsymbol{R}, t>0)$, wenn zwei Konstanten $E>0$ und $\varrho \geqq 0$ derart existieren, da $\beta$

$$
|P(k)| \geqq E|k|^{t}
$$

für alle $k \in Z^{n}$ mit $|k| \geqq \varrho$ gilt. (Wegen der Definition der $t$-Koerzitivität im ,,periodischen Fall" und wegen der äquivalenten Eigenschaft sei auf [8], S. 90, verwiesen.) Nach (5.2) gibt es ein $\varrho^{\prime} \geqq 0$ derart, daß

$$
|P(k)| \geqq 1
$$

für alle $k \in Z^{n}$ mit $|k| \geqq \varrho^{\prime}$ ist. Für diese $k$ hat man dann

$$
|P(k)| \geqq \frac{1}{\sqrt{2}}\left(1+|P(k)|^{2}\right)^{1 / 2}=\frac{1}{\sqrt{2}} \zeta_{*}(k) .
$$

Es sei noch bemerkt: Die Bedingung (5.2) der $t$-Koerzitivität ist der Forderung äquivalent, daß zu $P(D)$ zwei Konstanten $E^{\prime}>0$ und $\varrho^{\prime} \geqq 0$ existieren, mit denen

$$
|P(k)| \geqq E^{\prime}\left(1+|k|^{2}\right)^{t / 2}
$$

für alle $k \in Z^{n}$ mit $|k| \geqq \varrho^{\prime}$ gilt. Unsere Betrachtungen unterscheiden sich zu denjenigen von Louhivaara und Simader [8] dadurch, daß einerseits unsere Operatoren von allgemeineren Funktionen als Polynomen erzeugt werden können und andererseits die in Ungleichung (5.3) auftretende Zahl $t$ nicht mehr positiv zu sein braucht.

Weil es unter den $t$-koerzitiven Operatoren nichthypoelliptische gibt (man 
vergleiche Louhivaara und Simader [7], S. 17), gibt es auch nichthypoelliptische Operatoren $P(D)$, die $\zeta_{*}$-elliptisch $\left(\zeta_{*}(k)=\left(1+|P(k)|^{2}\right)^{1 / 2}\right)$ sind.

5.4. In 5.1 zeigten wir, daß jeder partielle Differentialoperator $P(D)$ mit konstanten Koeffizienten zur Klasse $\mathscr{A}^{1}\left(\zeta_{*}\right)$ gehört, mit $\zeta_{*}(k)=\left(1+|P(k)|^{2}\right)^{1 / 2}$. Jetzt wollen wir zeigen, daß auch eine bestimmte Klasse von Integraloperatoren in den hier betrachteten Rahmen behandelbar ist.

Sei $f \in L_{\text {per }}^{2}(Q)$ mit den Fourierkoeffizienten $f_{k}, k \in Z^{n}$. Man erkläre mit $\varphi \in S$ die Faltung

$$
F(x):=\int_{Q} f(x-\xi) \varphi(\xi) d \xi .
$$

Nur für endlich viele $l \in Z^{n}$ ist der Fourierkoeffizient $F_{l}$ von $F$ von Null verschieden, denn

$$
\begin{aligned}
F_{l} & =(2 \pi)^{-n / 2} \int_{Q} F(x) e^{-i(l, x)} d x=(2 \pi)^{-n / 2} \int_{Q}\left(\int_{Q} f(x-\xi) \varphi(\xi) d \xi\right) e^{-i(l, x)} d x \\
& =(2 \pi)^{-n} \sum_{\substack{k \in Z^{n} \\
\text { end. }}} \int_{Q}\left(\int_{Q} f(x-\xi) \varphi_{k} e^{i(k, \xi)-i(l, x)} d \xi\right) d x \\
& =(2 \pi)^{-n} \sum_{\substack{k \in Z^{n} \\
\text { endl. }}} \varphi_{k} \int_{Q}\left(\int_{Q} f(x-\xi) e^{-i(k, x-\xi)} d \xi\right) e^{i(k-l, x)} d x \\
& =(2 \pi)^{-n / 2} \sum_{\substack{k \in Z^{n} \\
\text { endl. }}} \varphi_{k} f_{k} \int_{Q} e^{i(k-l, x)} d x=(2 \pi)^{n / 2} \varphi_{l} f_{l} .
\end{aligned}
$$

Somit ist durch (5.4) ein Integraloperator

mit

$$
K(D): S \rightarrow S
$$

definiert. Wegen (5.5) gilt

$$
(K(D) \varphi)(x):=\int_{Q} f(x-\xi) \varphi(\xi) d \xi
$$

$$
(K(D) \varphi)(x)=-\sum_{k \in Z^{n}} \varphi_{k} f_{k} e^{i(k, x)} .
$$

Der Integraloperator $K(D)$ wird somit von der Abbildung

mit

$$
K: Z^{n} \rightarrow C
$$

$$
K(k):=(2 \pi)^{n / 2} f_{k} \quad \text { für alle } \quad k \in Z^{n}
$$

erzeugt. Wegen $|K(k)| \leqq(2 \pi)^{n / 2} \zeta_{*}(k)$, mit $\quad \zeta_{*}(k):=\left(1+\left|f_{k}\right|^{2}\right)^{1 / 2}$ folgt $K \in S^{1}\left(\zeta_{*}\right)$ und $K(D) \in \mathscr{A}^{1}\left(\zeta_{*}\right)$. 


\section{Wertemengen glättender Operatoren}

6.1. In 1.3 haben wir bemerkt, daß ein endlicher Operator $R(D): S \rightarrow S$ (für jedes $\left.\zeta_{*}\right) \zeta_{*}$-glättend ist, also in $\mathscr{A}^{-\infty}\left(\zeta_{*}\right)$ liegt. Weiter wurde in 4.3 erwähnt, daß die Fortsetzung $R_{r}(D)$ von $R(D)$ auf $H_{\zeta_{*}}^{r}(Q)$ auch endlich und (nach 3.1) $\zeta_{*}$ glättend in $H_{\zeta_{*}}^{r}(Q)$ ist, also in $\mathscr{A}_{r}^{-\infty}\left(\zeta_{*}\right)$ liegt.

Für die Fortsetzung $R_{r}(D)$ eines endlichen Operators $R(D)$ ist für jedes $u \in H_{\zeta_{*}}^{r}(Q)$ mit den Fourierkoeffizienten $u_{k}$ und mit $N=\left\{k \in Z^{n} \mid R(k) \neq 0\right\}$

$$
R_{r}(D) u(x)=(2 \pi)^{-n / 2} \sum_{k \in N} R(k) u_{k} e^{i(k, x)} .
$$

Somit ist $R_{r}(D) u$ fast überall gleich einer Funktion aus $C_{\text {per }}^{\infty}(Q)$, dafür schreiben wir

Folglich hat man

$$
R_{r}(D) u \in C_{\text {per }}^{\infty}(Q) \text {. }
$$

$$
R_{r}(D)\left(H_{\zeta_{*}}^{r}(Q)\right) \subset C_{\text {per }}^{\infty}(Q) .
$$

Diese Aussage läßt sich folgendermaßen verschärfen:

Lemma 6.1. Für die Gewichtsfunktion $\zeta_{*}$ und zu jedem $\alpha \in N_{0}^{n}$ existiere ein $t_{\alpha} \in \boldsymbol{R}$ derart, daß die Abschätzung

$$
\zeta_{*}^{t_{\alpha}}(k) \geqq C_{\alpha}\left|k^{\alpha}\right| \text { für alle } k \in Z^{n}
$$

gilt. Dann besteht die Relation (6.1) für jeden (in $H_{\zeta_{*}}^{r}(Q)$ ) $\zeta_{*}$-glättenden Operator $R_{r}(D)$.

Beweis. Da aufgrund des Sobolevschen Einbettungssatzes gilt

$$
\bigcap_{s \in R} H_{s}(Q) \subset C_{\mathrm{per}}^{\infty}(Q)
$$

(wegen der Definition von $H_{s}(Q)$ und dieses Resultates vergleiche man BersSchechter [1], S. 167), genügt es zu beweisen, daß für ein beliebiges Element $u \in H_{\zeta_{*}}^{r}(Q)$ und für jedes $\alpha \in N_{0}^{n}$ der Ausdruck $D^{\alpha}\left(R_{r}(D) u\right)$ ein Element aus $L_{\text {per }}^{2}(Q)$ erklärt. Dafür haben wir zu zeigen, daß zu $R_{r}(D) u$ eine Folge $\left\{\psi^{j}\right\}_{j \in N} \subset S$ existiert mit den beiden Eigenschaften:

1) Es gilt $\left\|R_{r}(D) u-\psi^{j}\right\|_{0} \rightarrow 0$ für $j \rightarrow \infty$.

2) Für jedes $\alpha \in N_{n}^{0}$ ist $\left\{D^{\alpha} \psi^{j}\right\}_{j \in N}$ eine Cauchyfolge in $L_{\text {per }}^{2}(Q)$.

Wegen $u \in H_{\zeta_{*}}^{r}(Q)$ gibt es eine Folge $\left\{\varphi^{j}\right\}_{j \in N} \subset S$ mit der Eigenschaft

$$
\left\|u-\varphi^{j}\right\|_{r, \zeta_{*}} \rightarrow 0 \text { für } j \rightarrow \infty .
$$

Aufgrund der Definition von $R(D)$ ist

$$
\psi^{j}:=R_{r}(D) \varphi^{j}=R(D) \varphi^{j}
$$

aus $S$, und wegen Lemma 3.1 hat man weiter

$$
\left\|R_{r}(D) u-\psi^{j}\right\|_{0} \rightarrow 0 \text { für } j \rightarrow \infty .
$$


Die soeben konstruierte Folge $\left\{\psi^{j}\right\}_{j \in N}$ hat aber auch die Eigenschaft 2). Zunächst ist

$$
\begin{aligned}
\left(D^{\alpha} \psi^{j}-D^{\alpha} \psi^{l}\right)(x) & =(2 \pi)^{-n \cdot 2} D^{\alpha}\left(\sum_{k \in Z^{n}} R(k)\left\{\left(\varphi^{j}\right)_{k}-\left(\varphi^{l}\right)_{k}\right\} e^{i(k, x)}\right) \\
& =(2 \pi)^{-n / 2} \sum_{k \in Z^{n}} R(k) k^{\alpha}\left\{\left(\varphi^{j}\right)_{k}-\left(\varphi^{l}\right)_{k}\right\} e^{i(k, x)},
\end{aligned}
$$

wobei die Summe endlich ist. Somit folgt wegen (6.2)

Da $R \in S^{-\infty}\left(\zeta_{*}\right)$, gilt gemäß (1.5)

$$
\begin{aligned}
\left\|D^{\alpha} \psi^{j}-D^{\alpha} \psi^{l}\right\|_{0}^{2} & =\sum_{k \in Z^{n}}|R(k)|^{2} k^{2 \alpha}\left|\left(\varphi^{j}\right)_{k}-\left(\varphi^{l}\right)_{k}\right|^{2} \\
& \leqq \frac{1}{C_{\alpha}^{2}} \sum_{k \in Z^{n}}|R(k)|^{2} \zeta_{*}^{2 t_{\alpha}}(k)\left|\left(\varphi^{j}\right)_{k}-\left(\varphi^{l}\right)_{k}\right|^{2} .
\end{aligned}
$$

und somit

$$
|R(k)| \leqq C \zeta_{*}^{r-t_{\alpha}}(k) \text { für alle } k \in Z^{n}
$$

$$
\left\|D^{\alpha} \psi^{j}-D^{\alpha} \psi^{l}\right\|_{0}^{2} \leqq C_{\alpha}^{\prime} \sum_{k \in Z^{n}} \zeta_{*}^{2 r}(k)\left|\left(\varphi^{j}\right)_{k}-\left(\varphi^{l}\right)_{k}\right|^{2} \leqq C_{\alpha}^{\prime}\left\|\varphi^{j}-\varphi^{l}\right\|_{r, \zeta_{*}}^{2}
$$

Im Falle $\zeta_{*}(k)=\left(1+|k|^{2}\right)^{1 / 2}$ fällt der Raum $H_{\zeta_{*}}^{r}(Q)$ mit dem Raum $H_{r}(Q)$ (aus Bers-Schechter [1], S. 167) zusammen, und Bedingung (6.2) ist erfüllt, denn es gilt für jedes $\alpha \in N_{0}$

$$
\left(\left(1+|k|^{2}\right)^{1 / 2}\right)^{|\alpha|} \geqq\left|k^{\alpha}\right| .
$$

Jetzt wollen wir eine Klasse von Abbildungen angeben, die als eine Erweiterung der Klasse der hypoelliptischen Polynome verstanden werden kann und für die Bedingung (6.2) gilt.

Lemma 6.2. Sei $\zeta$ eine Abbildung $\zeta: Z^{n} \rightarrow C$, die den folgenden Bedingungen genügt:

1) Es gibt $\alpha_{0} \in N_{0}^{n}, \alpha_{0} \neq 0$ und Zahlen $x \in \boldsymbol{R}, 0<\varkappa \leqq 1, C_{2} \geqq 0, \varrho \geqq 0$ derart, daß

$$
\frac{\left|D^{\alpha_{0}} \zeta(k)\right|}{|\zeta(k)|} \leqq C_{2}\left(1+|k|^{2}\right)^{-x\left|\alpha_{0}\right| / 2} \text { für alle } k \in Z^{n},|k|>\varrho
$$

gilt.

2) Zu obigem $\alpha_{0}$ gibt es Zahlen $r_{0} \in \boldsymbol{R}, C_{0}>0$ derart, daß

$$
\left|D^{\alpha_{0}} \zeta(k)\right| \geqq C_{0} \zeta_{*}^{r_{0}}(k) \text { für alle } k \in Z^{n},|k|>\varrho
$$

gilt.

Dann existieren zu jedem $\alpha \in N_{0}^{n}$ zwei Zahlen $t_{\alpha} \in R, C_{\alpha} \geqq 0$; mit denen Bedingung (6.2) für alle $k \in Z^{n}$ gilt.

Beweis. Wegen Voraussetzung 2) hat man

$$
\frac{\left|D^{\alpha_{0}} \zeta(k)\right|}{|\zeta(k)|} \geqq C_{0} \frac{\zeta_{*}^{r_{0}}(k)}{|\zeta(k)|} \geqq C_{0} \frac{\zeta_{*}^{r_{0}}(k)}{\left(1+|\zeta(k)|^{2}\right)^{1 / 2}} \geqq C_{0} \zeta_{*}^{r_{0}-1}(k)
$$

und weiter nach Voraussetzung 1)

$$
C_{2}\left(1+|k|^{2}\right)^{-\varkappa\left|\alpha_{0}\right| / 2} \geqq C_{0} \zeta_{*}^{r_{0}-1}(k)
$$


sowie

$$
\zeta_{*}^{1-r_{0}}(k) \geqq \frac{C_{0}}{C_{2}}\left(1+|k|^{2}\right)^{\varkappa\left|\alpha_{0}\right| / 2}
$$

für $|k|>\varrho$. Mit einer passenden neuen Konstanten $C_{1}>0$ erhält man die Abschätzung

$$
\zeta_{*}^{1-r_{0}}(k) \geqq C_{1}\left(1+|k|^{2}\right)^{x\left|\alpha_{0}\right| / 2} \quad \text { für alle } k \in Z^{n} .
$$

Für alle $\alpha \in N_{0}^{n}$ und für alle $k \in Z^{n}$ gilt zunächst

$$
\left|k^{\alpha}\right| \leqq\left(1+|k|^{2}\right)^{|\alpha| / 2}=\left(\left(1+|k|^{2}\right)^{x\left|\alpha_{0}\right| / 2}\right)^{|\alpha| /\left(x\left|\alpha_{0}\right|\right)}
$$

und weiter wegen (6.5)

$$
\left.\left(\zeta_{*}^{1-r_{0}}(k)\right)\right)^{|\alpha| /\left(x\left|\alpha_{0}\right|\right)} \geqq C_{1}^{|\alpha| /\left(x\left|\alpha_{0}\right|\right)}\left|k^{\alpha}\right| .
$$

Somit haben wir Ungleichung (6.2) mit $t_{\alpha}=\left(1-r_{0}\right)|\alpha| /\left(\varkappa\left|\alpha_{0}\right|\right)$ und $C_{\alpha}=C_{1}^{|\alpha| /\left(x\left|\alpha_{0}\right|\right)}$ bewiesen.

\section{Kompaktheit der Einbettung von $H_{\zeta_{*}}^{r}(Q)$ in $L_{\text {per }}^{2}(Q) \cdot \zeta_{*}$-Elliptizität}

\subsection{Wir zeigen :}

Satz 7.1. Es seien $r \in \boldsymbol{R}$ und $t \in \boldsymbol{R}, t>0$. Damit die Einbettung von $H_{\zeta_{*}}^{r+\boldsymbol{t}}(Q)$ in $H_{\zeta_{*}}^{r}(Q)$ kompakt ist, ist notwendig und hinreichend, daß

$$
\zeta_{*}(k) \rightarrow \infty \text { für } \quad|k| \rightarrow \infty
$$

gilt.

Beweis. A. Wir zeigen zunächst, daß aus (7.1) die Kompaktheit der Einbettung von $H_{\zeta_{*}}^{r+t}(Q)$ in $H_{\zeta_{*}}^{r}(Q)$ folgt.

Wir beginnen mit dem Beweis, daß die Menge

$$
M:=\left\{u \in S \mid\|u\|_{r+t, \zeta_{*}} \leqq 1\right\}
$$

in $H_{\zeta_{*}}^{r}(Q)$ präkompakt ist. Für jedes $m \in \bar{N}$ ist die Menge

$$
K_{m}:=\left\{k \in Z^{n} \mid \zeta_{*}(k)<m\right\}
$$

wegen (7.1) endlich. $\mathrm{Zu}$ einem beliebigen $u \in M$,

$$
u(x)=(2 \pi)^{-n / 2} \sum_{k \in Z^{n}} u_{k} e^{i(k, x)},
$$

erklären wir den ,,m-ten Abschnitt“" $u_{m}$ durch

Nun gilt

$$
u_{m}(x):=(2 \pi)^{-n / 2} \sum_{k \in K_{m}} u_{k} e^{i(k, x)} .
$$

$$
\left\|u-u_{m}\right\|_{r, \zeta_{*}}^{2}=\sum_{k \in Z^{n}-K_{m}} \zeta_{*}^{2 r}(k)\left|u_{k}\right|^{2},
$$


und wegen $\zeta_{*}(k) \geqq m$ für $k \in Z^{n}-K_{m}$ hat man

$$
\left\|u-u_{m}\right\|_{r, \zeta_{*}}^{2} \leqq \sum_{k \in Z^{n}-K_{m}} \xi_{*}^{2(r+t)}(k) \frac{1}{m^{2 t}}\left|u_{k}\right|^{2} \leqq \frac{1}{m^{2 t}}\|u\|_{r+t, \zeta_{*}}^{2} \leqq \frac{1}{m^{2 t}} .
$$

Somit bildet die Menge $M_{m}$ aller $m$-ten Abschnitte von Elementen aus $M$ für $m>(1 / \varepsilon)^{1 / t}$ in $H_{\zeta *}^{r}(Q)$ ein $\varepsilon$-Netz für die Menge $M$. Als eine beschränkte Menge in einem endlich dimensionalen Teilraum von $H_{\zeta_{*}}^{r}(Q)$ ist jedes $M_{m}$ präkompakt. Die Präkompaktheit des soeben konstruierten $\varepsilon$-Netzes ist wiederum hinreichend (und auch notwendig) für die Präkompaktheit von $M$ in $H_{\zeta_{*}}^{r}(Q)$. (Man vergleiche Triebel [11], S. 19-21.) Mithin ist die Einbettung von $H_{\zeta_{*}}^{r+t}(Q)$ in $H_{\zeta_{*}}^{r}(Q)$ kompakt.

B. Jetzt nehmen wir an, daß die Einbettung von $H_{\zeta_{*}}^{r+t}(Q)$ in $H_{\zeta_{*}}^{r}(Q)$ für je ein $r \in \boldsymbol{R}$ und $t \in \boldsymbol{R}, t>0$ kompakt ist. Wir werden zeigen, daß für eine beliebige Folge $\left\{k_{j}\right\}_{j \in N} \subset Z^{n}$ mit $\left|k_{j}\right| \rightarrow \infty$ die Aussage $\zeta_{*}\left(k_{j}\right) \rightarrow \infty$ gilt. Wir erklären die Funktion $u^{j}$ durch

$$
u^{j}(x):=\frac{1}{\zeta_{*}^{r+t}\left(k_{j}\right)} e^{i\left(k_{j}, x\right)}
$$

Für die Fourierkoeffizienten von $u^{j}$ hat man dann

und weiter

$$
\left(u^{j}\right)_{l}=(2 \pi)^{-n / 2} \frac{1}{\zeta_{*}^{r+t}\left(k_{j}\right)} \delta_{k_{j} l}
$$

$$
\left\|u^{j}\right\|_{r+t, \zeta_{*}}^{2}=\sum_{l \in Z^{n}}\left|\left(u^{j}\right)_{l}\right|^{2} \zeta_{*}^{2(r+t)}(l)=(2 \pi)^{-n}
$$

Somit ist die Folge $\left\{u^{j}\right\}_{j \in N}$ im Hilbertraum $H_{\zeta_{*}}^{r+t}(Q)$ beschränkt und enthält folglich eine schwach konvergente Teilfolge, die wir wieder mit $\left\{u^{j}\right\}_{j \in N}$ bezeichnen. Für jedes $\varphi \in S$ mit den Fourierkoeffizienten $\varphi_{l}$ ergibt sich

$$
\left(u^{j}, \varphi\right)_{r+t, \zeta_{*}}=\sum_{l \in Z^{n}} \zeta_{*}^{2(r+t)}(l) \overline{\left(u^{j}\right)_{l}} \varphi_{l}=(2 \pi)^{n / 2} \zeta_{*}^{r+t}\left(k_{j}\right) \varphi_{k_{j}} .
$$

Weil für ein festes $\varphi \in S$ der letztstehende Ausdruck für genügend große $k_{j}$ gleich Null ist, gilt trivialerweise

$$
\left(u^{j}, \varphi\right)_{r+t, \zeta_{*}} \rightarrow 0 \text { für } j \rightarrow \infty,
$$

d.h. die Folge $\left\{u^{j}\right\}_{j \in N}$ konvergiert in $H_{\zeta_{*}}^{r+t}(Q)$ schwach gegen Null. Wegen der Kompaktheit der Einbettung von $H_{\zeta_{*}}^{r+t}(Q)$ in $H_{\zeta_{*}}^{r}(Q)$ folgt $\left\|u^{j}\right\|_{r, \zeta_{*}} \rightarrow 0$ für $j \rightarrow \infty$. Wegen

hat man (mit $t>0$ )

$$
\left\|u^{j}\right\|_{r, \zeta_{*}}^{2}=\sum_{l \in \mathbb{Z}^{n}} \zeta_{*}^{2 r}(l)\left|\left(u^{j}\right)_{l}\right|^{2}=(2 \pi)^{-n} \frac{1}{\zeta_{*}^{2 t}\left(k_{j}\right)}
$$

$$
\frac{1}{\zeta_{*}^{t}\left(k_{j}\right)} \rightarrow 0 \text { für }\left|k_{j}\right| \rightarrow \infty .
$$

7.2. In dem Fall, daß die Einbettung $\imath: H_{\zeta *}^{r}(Q) \rightarrow L_{\text {per }}^{2}(Q)$ kompakt ist, geben wir ein zusätzliches Resultat an: 
Lemma 7.2. Es sei $\zeta: Z^{n} \rightarrow C$ eine beliebige Funktion und für ein $r \in \boldsymbol{R}, \boldsymbol{r}>0$, sei die Einbettung $\quad \imath: H_{\zeta_{*}}^{r}(Q) \rightarrow L_{\text {per }}^{2}(Q)$ kompakt. Dann gilt für jedes $R(D) \in \mathscr{A}^{-\infty}\left(\zeta_{*}\right)$ die Relation

$$
\lim _{|k| \rightarrow \infty}|R(k)|=0 .
$$

Beweis. Nach Satz 7.1 folgt aus der Kompaktheit der Einbettung , daß

$$
\lim _{|k| \rightarrow \infty} \frac{1}{\zeta_{*}^{r}(k)}=0
$$

ist. Nun läßt sich $R(D) \in \mathscr{A}^{-\infty}\left(\zeta_{*}\right)$ nach Lemma 3.1 für jedes $s \in \boldsymbol{R}$ stetig auf den Raum $H_{\zeta_{*}}^{s}(Q)$ fortsetzen. Folglich hat man für alle $t \in \boldsymbol{R}$ und alle $u \in H_{\zeta_{*}}^{s}(Q)$ die Ungleichung

$$
\left\|R_{s}(D) u\right\|_{(s-t), \zeta_{*}}^{2} \leqq C^{2}\|u\|_{s, \zeta_{*}}^{2} .
$$

Wählt man $s=0$ und $-t=r$, so gilt für alle $u \in L_{\text {per }}^{2}(Q)$

$$
\left\|R_{0}(D) u\right\|_{r, \zeta_{*}} \leqq C^{2}\|u\|_{0}^{2}
$$

beziehungsweise

$$
\sum_{k \in Z^{n}}|R(k)|^{2} \zeta_{*}^{2 r}(k)\left|u_{k}\right|^{2} \leqq C^{2} \sum_{k \in Z^{n}}\left|u_{k}\right|^{2} .
$$

Wählt man nun $u(x)=(2 \pi)^{-n / 2} e^{i(k, x)}$, so folgt für alle $k \in Z^{n}$

$$
|R(k)|^{2} \zeta_{*}^{2 r}(k) \leqq C^{2}
$$

oder

Somit gilt

$$
|R(k)| \leqq C \zeta_{*}^{-r}(k)
$$

$$
\lim _{|k| \rightarrow \infty}|R(k)| \leqq C \lim _{|k| \rightarrow \infty} \zeta_{*}^{-r}(k)=0 .
$$

7.3. Nun sind wir in der Lage, die folgende Charakterisierung der Kompaktheit der Einbettung $\quad l: H_{\zeta_{*}}^{r}(Q) \rightarrow L_{\text {per }}^{2}(Q)$ herzuleiten:

Satz 7.3. Es sei $\zeta: Z^{n} \rightarrow C$ eine beliebige Funktion. Damit aus der Existenz einer $\zeta_{*}$-Parametrix eines Operators $A(D) \in \mathscr{A}^{t}\left(\zeta_{*}\right)(t \in \boldsymbol{R})$ immer die $\zeta_{*}$-Elliptizität von $A(D)$ folgt, ist notwendig und hinreichend, daß die Einbettung $\quad \imath: H_{\zeta_{*}}^{r}(Q) \rightarrow L_{\mathrm{per}}^{2}(Q)$ für wenigstens ein $r \in \boldsymbol{R}, r>0$ kompakt ist.

Beweis. A. Die Einbettung $l$ sei kompakt. Der Operator $A(D) \in \mathscr{A}^{t}\left(\zeta_{*}\right)$ habe eine Parametrix $B(D) \in \mathscr{A}^{-t}\left(\zeta_{*}\right)$. Dann gibt es einen Operator $R(D) \in \mathscr{A}^{-\infty}\left(\zeta_{*}\right)$ mit

beziehungsweise

$$
B(D) \circ A(D)=I-R(D)
$$

$$
B(k) A(k)=1-R(k) \text { für alle } k \in Z^{n} .
$$

Da nach Lemma $7.2|R(k)| \rightarrow 0$ für $|k| \rightarrow \infty$ gilt, gibt es ein $\varrho \in \boldsymbol{R}, \varrho>0$ so, daß 
$1-|R(k)| \geqq 1 / 2$ für alle $k \in Z^{n}$ mit $|k|>\varrho$ ist. Für diese $k$ hat man daher

und folglich

$$
|B(k)||A(k)|=|1-R(k)| \geqq 1-|R(k)| \geqq \frac{1}{2}
$$

$$
|A(k)| \geqq \frac{1}{2|B(k)|} .
$$

Wegen $B(D) \in \mathscr{A}^{-t}\left(\zeta_{*}\right)$ gilt aber nach (1.5) mit einer passenden Konstanten $C$

Somit haben wir

$$
|B(k)| \leqq C \zeta_{*}^{-t}(k) \text { für alle } k \in Z^{n} .
$$

$$
|A(k)| \geqq \frac{1}{2 C} \zeta_{*}^{t}(k) \text { für alle } k \in Z^{n} \text { mit }|k|>\varrho,
$$

d.h. $A(D)$ ist $\zeta_{*}$-elliptisch.

B. Nun setzen wir voraus, daß die Einbettung $\imath: H_{\zeta_{*}}^{r}(Q) \rightarrow L_{\text {per }}^{2}(Q)$ für kein $r>0$ kompakt ist. Aus Satz 7.1 folgt, daß eine Folge $\left\{k_{j}^{\prime}\right\}_{j \in N}$ derart existiert, daß $\zeta_{*}\left(k_{j}^{\prime}\right)$ beschränkt bleibt. Dann gibt es aber eine Teilfolge $\left\{k_{j}\right\}_{j \in N}$, für die der Grenzwert

$$
\lim _{j \rightarrow \infty} \zeta_{*}\left(k_{j}\right)=: C^{\prime} \geqq 1
$$

existiert. Mit $K$ bezeichnen wir die von dieser Folge $\left\{k_{j}^{\prime}\right\}_{j \in N}$ gebildete Menge in $Z^{n}$. Man erkläre die Abbildung $R: Z^{n} \rightarrow C$ durch

$$
R(k)=\left\{\begin{array}{lll}
1 & \text { für } & k \in K, \\
0 & \text { für } & k \in Z^{n}-K
\end{array}\right.
$$

sowie den entsprechenden Operator $R(D): S \rightarrow S$.

Wegen (7.5) folgt die Existenz einer Zahl $\varrho>0$ derart, daß für alle $k \in K$ mit $|k|>\varrho$

$$
\frac{C^{\prime}}{2} \leqq \zeta_{*}(k) \leqq 2 C^{\prime}
$$

gilt. Mit $t>0$ ergibt sich für diese $k$

und mit $t<0$ entsprechend

$$
\left(\frac{2}{C^{\prime}}\right)^{t} \zeta_{*}^{t}(k) \geqq 1
$$

$$
\left(\frac{1}{2 C^{\prime}}\right)^{t} \zeta_{*}^{t}(k) \geqq 1 .
$$

Somit gilt für alle $t \in \boldsymbol{R}$ und alle $k \in K$ mit einer passenden von $t$ abhängigen Konstanten $C_{t}>0$

$$
R(k) \leqq C_{t} \zeta_{*}^{t}(k)
$$


(Man beachte, daß für $k \in Z^{n}-K$ obige Ungleichung trivialerweise gilt.) Also ist $R(D) \in \mathscr{A}^{-\infty}\left(\zeta_{*}\right)$.

Es sei nun $s \in \boldsymbol{R}$. Wir betrachten nun die durch

$$
A(k)=\left\{\begin{array}{lll}
0 & \text { für } & k \in K \\
\zeta_{*}^{s}(k) & \text { für } & k \in Z^{n}-K
\end{array}\right.
$$

erklärte Abbildung $A: Z^{n} \rightarrow C$ und den von $A$ induzierten Operator $A(D): S \rightarrow S$, der offenbar zu $\mathscr{A}^{s}\left(\zeta_{*}\right)$ gehört.

Ebenso erklären wir durch

$$
B(k)=\left\{\begin{array}{lll}
0 & \text { für } & k \in K \\
\zeta_{*}^{-s}(k) & \text { für } & k \in Z^{n}-K
\end{array}\right.
$$

die Abbildung $B: Z^{n} \rightarrow C$ sowie den durch $B$ induzierten Operator $B(D): S \rightarrow S$, der offenbar zu $\mathscr{A}^{-s}\left(\zeta_{*}\right)$ gehört.

Jetzt gilt für alle $k \in Z^{n}$

$$
B(k) A(k)=1-R(k)
$$

und somit

$$
B(D) \circ A(D)=I-R(D) \text {. }
$$

Also hat der Operator $A(D)\left(\in \mathscr{A}^{s}\left(\zeta_{*}\right)\right)$ eine Parametrix $B(D)\left(\in \mathscr{A}^{-s}\left(\zeta_{*}\right)\right)$, aber $A(D)$ ist nicht $\zeta_{*}$-elliptisch, weil $A(k)$ auch für gewisse $k$ mit beliebig großem $|k|$ verschwindet.

\section{Eine erweiterte Gårdingsche Ungleichung}

8.1. Wir zeigen nun für $\zeta_{*}$-elliptische Operatoren eine erweiterte Gårdingsche Ungleichung.

Satz 8.1. Sei $r \in \boldsymbol{R}$. Zu jedem $\zeta_{*}$-elliptischen Operator $A(D)$ aus $\mathscr{A}^{t}\left(\zeta_{*}\right)$ existieren zwei Konstanten $C_{0}>0$ und $C_{1} \geqq 0$ derart, daß die Ungleichung

$$
\|A(D) \varphi\|_{r, \zeta_{*}}^{2} \geqq C_{0}\|\varphi\|_{r+t, \zeta_{*}}^{2}-C_{1}\|\varphi\|_{r, \zeta_{*}}^{2}
$$

für alle $\varphi \in S$ gilt.

Beweis. Für $\varphi \in S$ mit den Fourierkoeffizienten $\varphi_{k}$ gilt nach (2.6) und (2.9)

$$
\|A(D) \varphi\|_{r, \zeta_{*}}^{2}=\sum_{k \in Z^{n}}|A(k)|^{2}\left|\varphi_{k}\right|^{2} \zeta_{*}^{2 r}(k)
$$

für jedes $r \in \boldsymbol{R}$. Aufgrund der $\zeta_{*}$-Elliptizität von $A(D)$ (man vergleiche (1.7)) hat man

$$
\begin{gathered}
\sum_{|k|>\varrho}|A(k)|^{2}\left|\varphi_{k}\right|^{2} \zeta_{*}^{2 r}(k) \geqq C \sum_{|k|>\varrho}\left|\varphi_{k}\right|^{2} \zeta_{*}^{2(r+t)}(k) \\
=C \sum_{k \in Z^{n}}\left|\varphi_{k}\right|^{2} \zeta_{*}^{2(r+t)}(k)-C \sum_{|k| \leqq \varrho}\left|\varphi_{k}\right|^{2} \zeta_{*}^{2(r+t)}(k)=C\|\varphi\|_{r+t, \zeta_{*}}^{2}-C \sum_{|k| \leqq \varrho}\left|\varphi_{k}\right|^{2} \zeta_{*}^{2(r+t)}(k) .
\end{gathered}
$$


Somit folgt

$$
\|A(D) \varphi\|_{r, \zeta_{*}}^{2} \geqq C\|\varphi\|_{r+t, \zeta_{*}}^{2}+\sum_{|k| \leqq \varrho}\left(|A(k)|^{2}-C \zeta_{*}^{2 t}(k)\right)\left|\varphi_{k}\right|^{2} \zeta_{*}^{2 r}(k) .
$$

Mit $C_{0}=C$ und $C_{1}=\left.\max _{|k|} \equiv_{\varrho}|| A(k)\right|^{2}-C \zeta_{*}^{2 t}(k) \mid$ folgt die Gültigkeit von (8.1).

Satz 8.2. Es seien $r, s \in \boldsymbol{R}$. Zu jedem $\zeta_{*}$-elliptischen Operator $A(D) \in \mathscr{A}^{t}\left(\zeta_{*}\right)$ existieren zwei Konstanten $C_{0}>0$ und $C_{s} \geqq 0 \quad\left(C_{s}\right.$ hängt von $s$ ab) derart, daß

für alle $\varphi \in S$ gilt.

$$
\|A(D) \varphi\|_{r, \zeta_{*}}^{2} \geqq C_{0}\|\varphi\|_{r+t, \zeta_{*}}^{2}-C_{s}\|\varphi\|_{s, \zeta_{*}}^{2}
$$

Beweis. Gemäß (8.2) gilt

$$
\begin{gathered}
\|A(D) \varphi\|_{r, \zeta_{*}}^{2} \geqq C\|\varphi\|_{r+t, \zeta_{*}}^{2}+\sum_{|k| \leqq \varrho}\left(|A(k)|^{2}-C \zeta_{*}^{2 t}(k)\right)\left|\varphi_{k}\right|^{2} \zeta_{*}^{2 r}(k) \\
\geqq C\|\varphi\|_{r+t, \zeta_{*}}^{2}+\sum_{|k| \leqq \varrho} \frac{|A(k)|^{2}-C \zeta_{*}^{2 t}(k)}{\zeta_{*}^{2(s-r)}(k)}\left|\varphi_{k}\right|^{2} \zeta_{*}^{2 s}(k) .
\end{gathered}
$$

Mit $C_{0}=C$ und

$$
C_{s}:=\max _{|k| \leqq \varrho} \frac{\left.|| A(k)\right|^{2}-C \zeta_{*}^{2 t}(k) \mid}{\zeta_{*}^{2(s-r)}(k)}
$$

folgt die Behauptung.

8.2. $\mathrm{Zu}$ Satz 8.2 gilt die folgende Umkehrung:

Satz 8.3. Man setze voraus, daß die Einbettung $\imath: H_{\zeta_{*}}^{r}(Q) \rightarrow L_{\mathrm{per}}^{2}(Q)$ für ein $r>0$ kompakt ist. Jeder Operator $A(D) \in \mathscr{A}^{t}\left(\zeta_{*}\right)$, der der Ungleichung (8.3) genügt, ist $\zeta_{*}$-elliptisch.

Beweis. Zu $k \in Z^{n}$ sei $\varphi^{k} \in S$ durch

$$
\varphi^{k}(x)=(2 \pi)^{-n / 2} e^{i(k, x)}
$$

erklärt. Aus (8.3) folgt dann für $s^{\prime} \in \boldsymbol{R}$

und damit

$$
|A(k)|^{2} \zeta_{*}^{2 r}(k) \geqq C_{0} \zeta_{*}^{2(r+t)}(k)-C_{1} \zeta_{*}^{2 s^{\prime}}(k)
$$

$$
|A(k)|^{2} \geqq C_{0} \zeta_{*}^{2 t}(k)-C_{1} \zeta_{*}^{2\left(s^{\prime}-r\right)}(k)
$$

für alle $k \in Z^{n}$. Da die Einbettung $\imath$ kompakt ist, folgt für jede reelle Zahl $s, s<r$, nach Satz 7.1

$$
\zeta_{*}^{2(s-r)}(k) \rightarrow 0 \text { für }|k| \rightarrow \infty .
$$

Also existiert eine reelle Zahl $\varrho \geqq 0$ derart, daß

$$
C_{1} \zeta_{*}^{2((s+t)-r)}(k) \leqq \frac{C_{0}}{2} \zeta_{*}^{2 t}(k)
$$


gilt, für alle $|k| \geqq \varrho$. Also folgt aus (8.4) mit $s^{\prime}=s+t$

$$
|A(k)|^{2} \geqq C_{0}^{2 t} \zeta_{*}^{2 t}(k)-\frac{C_{0}}{2} \zeta_{*}^{2 t}(k)
$$

und somit

$$
|A(k)| \geqq\left(\frac{C_{0}}{2}\right)^{1 / 2} \zeta_{*}^{t}(k),|k| \geqq \varrho .
$$

\section{Literatur}

[1] Bers, L., und M. ScheChter: Elliptic equations. - Partial differential equations (by L. Bers, F. John, M. Schechter). Lectures in applied mathematics (Proceedings of the summer seminar, Boulder, Colorado, 1957) III. Interscience Publishers, a division of John Wiley \& Sons, Inc., New York-London-Sydney, 1964, 131-299.

[2] EelLS, J.: Elliptic operators on manifolds. - Complex analysis and its applications (Lectures presented at an international seminar course at Trieste, 1975) I. International Centre for Theoretical Physics, Trieste. International Atomic Energy Agency, Vienna, 1976, 95-152.

[3] Gajewski, H., K. GröGer und K. Zacharias: Nichtlineare Operatorengleichungen und Operatorendifferentialgleichungen. - Mathematische Monographien 38. AkademieVerlag, Berlin, 1974.

[4] Hörmander, L.: Linear partial differential operators. - Die Grundlehren der mathematischen Wissenschaften 116. Springer-Verlag, Berlin-Göttingen-Heidelberg, 1963.

[5] Kohn, J. J., und L. Nirenberg: An algebra of pseudodifferential operators. - Comm. Pure Appl. Math. 18, 1965, 269-305.

[6] LAX, P. D.: On Cauchy's problem for hyperbolic equations and the differentiability of solutions of elliptic equations. - Comm. Pure Appl. Math. 8, 1955, 615-633.

[7] LouhivaAra, I. S., und C. G. Simader: Über nichtelliptische lineare partielle Differentialoperatoren mit konstanten Koeffizienten. - Ann. Acad. Sci. Fenn. Ser. A. I. Math. $513,1972$.

[8] LouhivaAra, I. S., und C. G. Simader: Über periodische Lösungen koerzitiver linearer partieller Differentialgleichungen. - Ann. Acad. Sci. Fenn. Ser. A. I. Math. 4, 1978/79, 87-108.

[9] TAYLOR, M.: Pseudodifferential operators. - Princeton Mathematical Series 34. Princeton University Press, Princeton (New Jersey), 1981.

[10] TRÈves, F.: Linear partial differential equations with constant coefficients. - Gordon \& Breach, New York, 1966.

[11] Triebel, H.: Höhere Analysis. - Hochschulbücher für Mathematik 76. Deutscher Verlag der Wissenschaften, Berlin, 1976.

Freie Universität Berlin

Fachbereich Mathematik

Arnimallee 2-6

D 1000 Berlin 33

Bundesrepublik Deutschland

Eingegangen am 3. August 1982
Neue Arschrift von N. J.:

Hochschule der Bundeswehr München

Fachbereich Informatik

Institut für Mathematik

Werner-Heisenberg-Weg 39

D 8014 Neubiberg

Bundesrepublik Deutschland 